\title{
EIGENVALUE PROBLEMS FOR ONE-DIMENSIONAL DISCRETE SCHRÖDINGER OPERATORS WITH SYMMETRIC BOUNDARY CONDITIONS*
}

\author{
JONQ JUANG ${ }^{\dagger}$, WEN-WEI LIN`, AND SHIH-FENG SHIEH ${ }^{\dagger}$
}

\begin{abstract}
In this paper, we investigate the one-dimensional discrete Schrödinger equation with general, symmetric boundary conditions. Our results primarily concern the number of energy states lying in the wells.
\end{abstract}

Key words. eigenvalue, Schrödinger operator, boundary conditions

AMS subject classifications. Primary, 34L15, 34L40; Secondary, 81Q10, 34B05

PII. S0895479800371530

1. Introduction. Allowing $a>0$, we consider a discrete version of the eigenvalue problem of the Schrödinger operator

$$
-\left(\frac{1}{m(x)} u^{\prime}\right)^{\prime}+V(x) u=\lambda u
$$

on a finite interval $I=[-a, a]$. Here $V(x)$ denotes the quantum well potential defined by

$$
V(x)= \begin{cases}-v, & x \in[-b, b], \\ 0 & \text { otherwise }\end{cases}
$$

where $a>b>0$ and $v>0$. Moreover, $m(x)$ is assumed to be a piecewise constant function. Specifically,

$$
m(x)= \begin{cases}m^{\prime}, & x \in[-b, b] \\ m & \text { otherwise }\end{cases}
$$

To discretize (1.1a), we divide $[-a, a]$ into equal parts of length $h$ and assume $a$ and $b$ are integer multiples of $h$, i.e., $a=(M+N+1) h$ and $b=N h$. The node points of the discrete equation are

$$
x_{i}=\left(\frac{a}{N+M+1}\right) i, \quad-M-N-1 \leq i \leq M+N+1 .
$$

\footnotetext{
${ }^{*}$ Received by the editors May 2, 2000; accepted for publication (in revised form) by P. Van Dooren June 14, 2001; published electronically November 13, 2001.

http://www.siam.org/journals/simax/23-2/37153.html

${ }^{\dagger}$ Department of Applied Mathematics, National Chiao Tung University, Taiwan (jjuang@math. tamu.edu, ssf@math.nctu.edu.tw). The first and third authors completed their research while on sabbatical at Texas A\&M University.

${ }^{\ddagger}$ Department of Mathematics, National Tsing Hua University, Hsin-Chu 30050, Taiwan (wwlin@ am.nthu.edu.tw).
} 
Using a standard central-differencing technique, we then obtain the discrete version of (1.1) as follows:

$$
\begin{aligned}
& u_{i+1}-2\left(1-m^{\prime}(\lambda+v)\right) u_{i}+u_{i-1}=0, \quad-N+1 \leq i \leq N-1, \\
& u_{i+1}-2(1-m \lambda) u_{i}+u_{i-1}=0, \quad N+2 \leq|i| \leq N+M, \\
& \frac{u_{N+1}}{m+m^{\prime}}+\frac{u_{N-1}}{2 m^{\prime}}-\frac{u_{N}}{m+m^{\prime}}-\frac{u_{N}}{2 m^{\prime}}+(\lambda+v) u_{N}=0, \\
& \frac{u_{N+2}}{2 m}+\frac{u_{N}}{m+m^{\prime}}-\frac{u_{N+1}}{2 m}-\frac{u_{N+1}}{m+m^{\prime}}+\lambda u_{N+1}=0, \\
& \frac{u_{-N-1}}{m+m^{\prime}}+\frac{u_{-N+1}}{2 m^{\prime}}-\frac{u_{-N}}{m+m^{\prime}}-\frac{u_{-N}}{2 m^{\prime}}+(\lambda+v) u_{-N}=0,
\end{aligned}
$$

and

$$
\frac{u_{-N-2}}{2 m}+\frac{u_{-N}}{m+m^{\prime}}-\frac{u_{-N-1}}{2 m}-\frac{u_{-N-1}}{m+m^{\prime}}+\lambda u_{-N-1}=0 .
$$

We remark that the discrete formulation (1.2) of (1.1a) has been normalized in such a way that the step size $h$ of the discretization is absorbed in $\lambda$ and $v$. The following "symmetric" general boundary conditions are imposed:

$$
u_{N+M+1}=\beta u_{-(N+M)}+\gamma u_{(N+M)}
$$

and

$$
u_{-(N+M+1)}=\beta u_{(N+M)}+\gamma u_{-(N+M)} .
$$

In particular, $\beta=0$ and $\gamma=1$ (resp., $\gamma=0$ and $\beta=1$ ) correspond to Neumann (resp., periodic) boundary conditions.

Eigenvalue ratios and gaps for the Schrödinger operators have been investigated by many authors (see, e.g., $[1,2,5,9,10]$ and the works cited therein). On the other hand, the number $A(d)$ of eigenvalues less than a bound $d$ is also of interest. Some partial results concerning the asymptotic behavior (i.e., as $d \rightarrow \infty$ ) of $A(d)$ are contained in [3]. We are led to investigate in this paper the number of energy states (eigenvalues) for a discrete Schrödinger problem (1.2a)-(1.2h) lying in the wells by the following work. In $[6,8]$, the spatial tunneling (from one well to the other) occurs in coupled quantum wells when the energy states in both wells are aligned. In the case of the hole tunneling in the coupled quantum wells, the tunneling mechanisms are significantly complicated due to band mixing effects. When the energy states are approximately aligned between heavy hole and light hole, the mixing tunneling occurs. Moreover, it was reported in [7] that the chaotic tunneling effect was generated when these two tunneling effects have a strong interaction between them. Our effort here is the first step toward understanding those phenomena.

Our main results are recorded in section 2 . Specifically, we construct the characteristic equations of the problem (1.2a)-(1.2h). By analyzing the properties of such equations, we are able to compute the number of energy states in the well for some general, symmetric boundary conditions.

2. The main results. We begin with the following definition.

DEFINITION 2.1. An eigenvector $\left(u_{i}\right)_{i=-(N+M+1)}^{N+M+1}$ of $(1.2 \mathrm{a})-(1.2 \mathrm{~h})$ is said to be symmetric (resp., antisymmetric) if $u_{i}=u_{-i}$ (resp., $\left.u_{i}=-u_{-i}\right)$. 
Proposition 2.1. Each eigenvector of (1.2a)-(1.2h) is either symmetric or antisymmetric.

Proof. It follows from the rank one modification of the symmetric matrices (see, e.g., [4]) that every eigenvalue of (1.2a) $-(1.2 \mathrm{~h})$ is simple. Let $\left(u_{i}\right)_{i=-(N+M+1)}^{N+M+1}$ be an eigenvector corresponding to some eigenvalue $\lambda$. Note that $\left(u_{-i}\right)_{i=-(N+M+1)}^{N+M+1}$ is also an eigenvector associated with $\lambda$. Thus, $u_{i}+u_{-i}$ and $u_{i}-u_{-i}$ are in the eigenspace corresponding to $\lambda$. We see that $u_{i}+u_{-i}$ is symmetric and $u_{i}-u_{-i}$ is antisymmetric. The assertion of the proposition now follows from the facts that every eigenspace is one-dimensional and that one of the vectors $u_{i}+u_{-i}$ and $u_{i}-u_{-i}$ is nonzero.

DeFINITION 2.2. Let $\lambda$ be an eigenvalue of $(1.2 \mathrm{a})-(1.2 \mathrm{~h})$ whose corresponding eigenvector is symmetric (resp., antisymmetric); then $\lambda$ is said to be symmetric (resp., antisymmetric).

We remark that Definition 2.2 is well defined since every eigenspace is onedimensional. We next derive some "characteristic" equations whose roots are eigenvalues of the system (1.2a)-(1.2h). To this end, we first assume $\lambda$ is a symmetric eigenvalue; then

$$
u_{i+1}-2\left(1-m^{\prime}(v+\lambda)\right) u_{i}+u_{i-1}=0 \quad \text { for } \quad|i| \leq N-1 .
$$

Hence,

$$
u_{i}=A\left(s^{i}+s^{-i}\right) \quad \text { for } \quad|i| \leq N,
$$

where $A$ is a constant to be determined and

$$
s=1-m^{\prime}(v+\lambda)+\sqrt{\left(1-m^{\prime}(v+\lambda)\right)^{2}-1}
$$

is a root of the characteristic polynomial $x^{2}-2\left(1-m^{\prime}(v+\lambda)\right) x+1=0$. In particular, for

$$
-v+\frac{2}{m^{\prime}} \geq \lambda \geq-v
$$

we see that

$$
u_{i}=2 A \cos i \theta .
$$

Here $\theta=\cos ^{-1}\left(1-m^{\prime}(v+\lambda)\right)$. For $N+2 \leq|i| \leq N+M$,

$$
u_{i+1}-2(1-m \lambda) u_{i}+u_{i-1}=0,
$$

and we have that

$$
u_{i}= \begin{cases}B t^{i-N}+C t^{-(i-N)}, & N+1 \leq i \leq N+M+1, \\ B t^{-(i+N)}+C t^{i+N}, & -(N+M+1) \leq i \leq-(N+1) .\end{cases}
$$

Here $B$ and $C$ are constants to be determined and

$$
t=(1-m \lambda)+\sqrt{(1-m \lambda)^{2}-1}
$$

Using the boundary condition $(1.2 \mathrm{~g})$ and the fact that $u$ is symmetric, we see that

$$
u_{N+M+1}=(\beta+\gamma) u_{N+M} .
$$


Applying (2.5a) to (2.7), we get

$$
\begin{aligned}
C & =\left(t^{2 M+1}\right)\left(\frac{\beta+\gamma-t}{1-(\beta+\gamma) t}\right) B \\
& :=\left(t^{2 M+1}\right)\left(D_{\beta}\right) B .
\end{aligned}
$$

Hence,

$$
u_{i}=B\left(t^{i-N}+t^{2 M+1}\left(D_{\beta}\right) t^{-(i-N)}\right), \quad N+1 \leq i \leq N+M+1 .
$$

We next consider the connection at $i=N$ and $i=N+1$. Let

$$
\alpha=\frac{2 m}{m+m^{\prime}} \quad \text { and } \quad \alpha^{\prime}=\frac{2 m^{\prime}}{m+m^{\prime}}
$$

at $i=N$; we thus write $(1.2 \mathrm{c})$ as

$$
\alpha^{\prime} u_{N+1}+\left(1-\alpha^{\prime}\right) u_{N}-2\left(1-m^{\prime}(\lambda+v)\right) u_{N}+u_{N-1}=0 .
$$

Using (2.11), (2.1), and (2.8), we see that (2.11) reduces to

$$
\begin{aligned}
& A\left(1-\alpha^{\prime}\right)\left(s^{N}+s^{-N}\right)+A\left(-2\left(1-m^{\prime}(\lambda+v)\right)\left(s^{N}+s^{-N}\right)\right)+A\left(s^{N-1}+s^{-N+1}\right) \\
& =-\alpha^{\prime} B\left(t+\left(D_{\beta}\right) t^{2 M}\right) .
\end{aligned}
$$

Noting that $s^{N}$ and $s^{-N}$ satisfy the recursive relation as given in (1.2a) with $i=N$, we see that $(2.11)$ reduces to

$$
A\left[\left(\alpha^{\prime}-1\right)\left(s^{N}+s^{-N}\right)+\left(s^{N+1}+s^{-(N+1)}\right)\right]=\alpha^{\prime} B\left(t+\left(D_{\beta}\right) t^{2 M}\right) .
$$

At $i=N+1$, a similar process yields

$$
A \alpha\left(s^{N}+s^{-N}\right)=B\left[(\alpha-1)\left(t+\left(D_{\beta}\right) t^{2 M}\right)+\left(1+\left(D_{\beta}\right) t^{2 M+1}\right)\right] .
$$

Dividing (2.13) by (2.14), we conclude that every symmetric eigenvalue is a root of

$$
\left[\frac{\alpha-1}{\alpha^{\prime}}+\frac{1+\left(D_{\beta}\right) t^{2 M+1}}{\alpha^{\prime}\left(t+\left(D_{\beta}\right) t^{2 M}\right)}\right]^{-1}=\frac{\alpha^{\prime}-1}{\alpha}+\frac{1}{\alpha} \frac{s^{N+1}+s^{-(N+1)}}{s^{N}+s^{-N}},
$$

where $D_{\beta}$ is defined in (2.8). Similarly, we obtain that every antisymmetric eigenvalue is a root of

$$
\left[\frac{\alpha-1}{\alpha^{\prime}}+\frac{1+\left(D_{-\beta}\right) t^{2 M+1}}{\alpha^{\prime}\left(t+\left(D_{-\beta}\right) t^{2 M}\right)}\right]^{-1}=\frac{\alpha^{\prime}-1}{\alpha}+\frac{1}{\alpha} \frac{s^{N+1}-s^{-(N+1)}}{s^{N}-s^{-N}} .
$$

To investigate (2.15) and (2.16), we need to set up the following notations:

$$
\begin{aligned}
\xi_{i}^{ \pm} & :=\frac{1+\left(D_{ \pm \beta}\right) t^{2 i+1}}{t+\left(D_{ \pm \beta}\right) t^{2 i}}=\frac{t^{-i}+\left(D_{ \pm \beta}\right) t^{i+1}}{t^{1-i}+\left(D_{ \pm \beta}\right) t^{i}}, \\
\eta_{i}^{ \pm} & :=\frac{s^{i+1} \pm s^{-(i+1)}}{s^{i} \pm s^{-i}} \\
f^{ \pm}(\lambda) & =\frac{\alpha^{\prime}}{(\alpha-1)+\xi_{M}^{ \pm}}
\end{aligned}
$$


and

$$
g^{ \pm}(\lambda)=\frac{\alpha^{\prime}-1}{\alpha}+\frac{\eta_{N}^{ \pm}}{\alpha}
$$

Now (2.15) and (2.16) can be written as

$$
f^{+}(\lambda)=g^{+}(\lambda)
$$

and

$$
f^{-}(\lambda)=g^{-}(\lambda)
$$

DEFINITION 2.3. Equations (2.21) and (2.22) are called the symmetric and antisymmetric characteristic equations of system $(1.2 \mathrm{a})-(1.2 \mathrm{~h})$.

Obviously, the roots of symmetric (resp., antisymmetric) characteristic equations are the symmetric (resp., antisymmetric) eigenvalues of system (1.2a)-(1.2h). The following useful recursive formulas can be verified directly:

$$
\begin{aligned}
\xi_{i+1}^{ \pm} & =2(1-m \lambda)-\frac{1}{\xi_{i}^{ \pm}}, \quad 0 \leq i \leq M-1, \\
\xi_{0}^{ \pm} & =\frac{1}{\gamma \pm \beta},
\end{aligned}
$$

and

$$
\begin{aligned}
\eta_{i+1}^{ \pm} & =2\left(1-m^{\prime}(v+\lambda)\right)-\frac{1}{\eta_{i}^{ \pm}}, \quad 0 \leq i \leq N-1, \\
\eta_{0}^{+} & =1-m^{\prime}(v+\lambda), \quad \eta_{0}^{-}=\infty .
\end{aligned}
$$

In the following, we shall study the properties of functions $f^{ \pm}$and $g^{ \pm}$.

Proposition 2.2. For whatever $\lambda$ is defined, $\frac{d}{d \lambda} f^{ \pm}(\lambda)>0$ and $\frac{d}{d \lambda} g^{ \pm}(\lambda)<0$.

Proof. We illustrate only the proof of $\frac{d}{d \lambda} g^{+}(\lambda)<0$. The rest is similar. Using (2.24) we see that

$$
\frac{d}{d \lambda} \eta_{i+1}^{+}=-2 m^{\prime}+\frac{1}{\left(\eta_{i}^{+}\right)^{2}} \frac{d}{d \lambda} \eta_{i}^{+}
$$

and

$$
\frac{d}{d \lambda} \eta_{0}^{+}=-m^{\prime}<0
$$

By induction, we conclude that $\frac{d}{d \lambda} \eta_{N}^{+}<0$ and, hence, that $\frac{d}{d \lambda} g^{+}(\lambda)<0$.

To further study $f^{ \pm}$and $g^{ \pm}$, we need the following map:

$$
F_{\lambda, m}(\xi)=2(1-m \lambda)-\frac{1}{\xi} .
$$

Proposition 2.3. The following assertions hold true:

(i) $F_{\lambda, m}$ maps $[1, \infty] \cup[-\infty, 0)$ into $[1, \infty)$ for $\lambda \in(-\infty, 0]$.

(ii) $F_{\lambda, m}$ maps $[-\infty,-1] \cup(0, \infty]$ into $(-\infty,-1]$ for $\lambda \in\left[\frac{2}{m}, \infty\right)$.

(iii) $F_{\lambda, m}(\xi)$ is strictly increasing on $(-\infty, 0)$ and on $(0, \infty)$ for any $\lambda$. 
We skip the proof of Proposition 2.3.

Proposition 2.4. Let $2>\alpha>0$. If $\gamma+\beta \leq 1$ (resp., $\gamma-\beta \leq 1$ ), then $f^{+}(\lambda)$ (resp., $f^{-}(\lambda)$ ) is continuous on $(-\infty, 0]$. Moreover, $g^{ \pm}(\lambda)$ are continuous on $(-\infty,-v] \cup\left[-v+\frac{2}{m^{\prime}}, \infty\right)$. If $-1 \leq \gamma+\beta$ (resp., $\left.-1 \leq \gamma-\beta\right)$, then $f^{+}(\lambda)\left(\right.$ resp.,$\left.f^{-}(\lambda)\right)$ is continuous on $\left[\frac{2}{m}, \infty\right)$.

Proof. Let $\lambda \in(-\infty, 0]$. If $\gamma \pm \beta \leq 1$, then $\xi_{0}^{ \pm} \in[1, \infty)$ or $(-\infty, 0)$. Suppose $\xi_{0}^{ \pm} \in[1, \infty)$. Then it follows from Proposition 2.3(i) that

$$
\xi_{M}^{ \pm}(\lambda)=F_{\lambda, m}^{M}\left(\xi_{0}^{ \pm}\right) \in[1, \infty) .
$$

Hence $-1+\alpha+\xi_{M}^{ \pm}(\lambda) \neq 0$, and so $f^{ \pm}$is continuous on $(-\infty, 0]$.

Suppose $\lambda \in(-\infty,-v] \cup\left[-v+\frac{2}{m^{\prime}}, \infty\right)$. Then $\eta_{0}^{-}, \eta_{0}^{+}=1-m^{\prime}(v+\lambda) \in[1, \infty] \cup$ $[-\infty,-1]$. It then follows from Proposition 2.3(i)-(ii) that

$$
\eta_{N}^{ \pm}(\lambda)=F_{\lambda+v, m^{\prime}}^{N}\left(\eta_{0}^{ \pm}\right) \in(-\infty,-1] \cup[1, \infty) .
$$

Hence, $g^{ \pm}(\lambda)$ is continuous on $(-\infty,-v] \cup\left[-v+\frac{2}{m^{\prime}}, \infty\right)$. If $-1 \leq \gamma+\beta$ and $\lambda$ is in $\left[\frac{2}{m}, \infty\right)$, then $F_{\lambda, m}\left(\xi_{0}^{+}\right) \leq-2-(\gamma+\beta) \leq-1$. Hence, $\xi_{M}^{+}(\lambda)=F_{\lambda, m}^{M}\left(\xi_{0}^{+}\right) \in(-\infty,-1]$. The case for $-1 \leq \gamma-\beta$ can be similarly obtained. We thus complete the proof of the proposition.

It is clear from Proposition 2.4 that the singularities of $g^{ \pm}(\lambda)$ occur in $\left(-v,-v+\frac{2}{m^{\prime}}\right)$, and that, for $-1 \leq \gamma+\beta \leq 1$, the singularities of $f^{ \pm}(\lambda)$ stay in $\left(0, \frac{2}{m}\right)$.

Proposition 2.5. (i) For $\lambda \in\left(-v,-v+\frac{2}{m^{\prime}}\right), g^{+}(\lambda)$, respectively, $g^{-}(\lambda)$, has singularities at

$$
-v+\frac{1}{m^{\prime}}\left(1-\cos \frac{2 k-1}{2 N} \pi\right)=: d_{k}, \quad i=1,2, \ldots, N,
$$

respectively,

$$
-v+\frac{1}{m^{\prime}}\left(1-\cos \frac{k}{N} \pi\right)=: e_{k}, \quad k=1,2, \ldots, N-1 .
$$

(ii) The following ordering holds true:

$$
-v<d_{1}<e_{1}<d_{2}<e_{2}<\cdots<e_{n-1}<d_{n}<e_{N}:=-v+\frac{2}{m^{\prime}} .
$$

Proof. The proof of Proposition 2.5(i) follows from (2.18). The ordering in (2.27c) is obvious.

Our main concern in this paper is the number of energy levels (eigenvalues) falling in the well, that is, the number of eigenvalues whose value is no greater than zero. Hence, the characterization of the singularities of $f^{ \pm}(\lambda)$ on $\left(0, \frac{2}{m}\right)$ will not be pursued here.

Proposition 2.6. (i) Let $0<\alpha<2$. If $\gamma \pm \beta \leq 1$, then

$$
\frac{\alpha^{\prime}}{\alpha+1}<f^{ \pm}(0)=\frac{\alpha^{\prime}}{(\alpha-1)+\frac{1-(\gamma \pm \beta-1) M}{\gamma \pm \beta-(\gamma \pm \beta-1) M}} \leq \frac{\alpha^{\prime}}{\alpha} .
$$

Consequently, $f^{ \pm}(-v)<\frac{\alpha^{\prime}}{\alpha}$. (ii) $g^{+}(-v)=\frac{\alpha^{\prime}}{\alpha}, \frac{\alpha+1}{\alpha}>g^{-}(-v)>\frac{\alpha^{\prime}}{\alpha}$. (iii) If $v>\frac{2}{m^{\prime}}$, then $g^{ \pm}(0)<\frac{\alpha^{\prime}-2}{\alpha}$. 
Proof. It follows from l'Hôspital's rule that

$$
\lim _{\lambda \rightarrow 0} \xi_{M}^{ \pm}=\lim _{t \rightarrow 1} \frac{1+\frac{(\gamma \pm \beta)-t}{1-(\gamma \pm \beta) t} t^{2 M+1}}{t+\frac{(\gamma \pm \beta)-t}{1-(\gamma \pm \beta) t} t^{2 M}}=\frac{1-(\gamma \pm \beta-1) M}{\gamma \pm \beta-(\gamma \pm \beta-1) M} .
$$

To see the estimates for $\xi_{M}^{ \pm}$at $\lambda=0$, we consider the map $F_{0, m}$, as defined in (2.25). It is then clear that $1 \leq \xi_{M}^{ \pm}<2$. Hence, $\frac{\alpha^{\prime}}{\alpha+1}<f^{ \pm}(0) \leq \frac{\alpha^{\prime}}{\alpha}$ as claimed. Since $f^{ \pm}$ is increasing on $(-\infty, 0]$, we obtain that $f^{ \pm}(-v)<\frac{\alpha^{\prime}}{\alpha}$. The assertions in (ii) are trivial. If $v>\frac{2}{m^{\prime}}$, then, for $\lambda=0, \eta_{0}=1-m^{\prime} v \leq-1$. Using $F_{v, m^{\prime}}$, we see, via Proposition 2.3(ii), that $\eta_{N}^{+}(0) \leq-1$, and, hence, $g^{+}(0) \leq \frac{\alpha^{\prime}-2}{\alpha}$. Similarly, we obtain that $g^{-}(0) \leq \frac{\alpha^{\prime}-2}{\alpha}$.

Notation 2.1. Set $R_{1}=\{(\gamma, \beta): \beta \leq 0, \gamma-\beta \leq 1\}$ and $R_{2}=\{(\gamma, \beta): \beta \geq 0$, $\gamma+\beta \leq 1\}$

Proposition 2.7. Let $2>\alpha>0$. If $(\gamma, \beta) \in R_{1}$ (resp., $\left.(\gamma, \beta) \in R_{2}\right)$, then $f^{-}(\lambda) \geq f^{+}(\lambda)$ (resp., $f^{-}(\lambda) \leq f^{+}(\lambda)$ ) on $(-\infty, 0]$. The equality holds only if $\beta=0$.

Proof. We first note that $R_{1} \cup R_{2}=\{(\gamma, \beta): \gamma+\beta \leq 1$ and $\gamma-\beta \leq 1\}$. Let $(\gamma, \beta) \in R_{1}$; then one of the following three cases holds:

$$
\xi_{0}^{+} \geq \xi_{0}^{-} \geq 1, \quad 0>\xi_{0}^{+} \geq \xi_{0}^{-}, \quad \text { and } \quad \xi_{0}^{-}>1>0>\xi_{0}^{+} .
$$

Furthermore, if the order of $\xi_{0}^{+}$and $\xi_{0}^{-}$satisfies one of the three cases in (2.28), then $F_{\lambda, m}\left(\xi_{0}^{+}\right) \geq F_{\lambda, m}\left(\xi_{0}^{-}\right) \geq 1$. It then follows from Proposition 2.5(ii)-(iii) that $\xi_{M}^{+}(\lambda)=F_{\lambda, m}^{M}\left(\xi_{0}^{+}\right) \geq F_{\lambda, m}^{M}\left(\xi_{0}^{-}\right)=\xi_{M}^{-}(\lambda)$. Consequently, $f^{+}(\lambda) \leq f^{-}(\lambda)$. The other case can be similarly obtained. It is clear that $f^{-}(\lambda)=f^{+}(\lambda)$ only if $\xi_{0}^{+}=\xi_{0}^{-}$or, equivalently, $\beta=0$.

Proposition 2.8. (i) $g^{+}(\lambda)<g^{-}(\lambda)$ for $\lambda \in\left(-\infty, d_{1}\right) \cup \bigcup_{i=1}^{N-1}\left(e_{i}, d_{i+1}\right)$.

(ii) $g^{+}(\lambda)>g^{-}(\lambda)$ for $\lambda \in \bigcup_{i=1}^{N}\left(d_{i}, e_{i}\right) \cup\left[e_{N}, \infty\right)$.

Proof. For $\lambda \in(-\infty,-v], \eta_{0}^{+}=1-m^{\prime}(v+\lambda) \geq 1$. It follows from Proposition 2.3(i) that $F_{v+\lambda, m^{\prime}}(\xi) \geq 1$ for $\xi=\eta_{0}^{+}$or $\eta_{0}^{-}$. Hence, we see, via Proposition 2.3(iii), that $g^{+}(\lambda)<g^{-}(\lambda)$. It is clear that

$$
\lim _{\lambda \rightarrow d_{i}^{-}} g^{+}(\lambda)=-\infty, \lim _{\lambda \rightarrow d_{i}^{+}} g^{+}(\lambda)=+\infty, \quad i=1,2, \ldots, N,
$$

and

$$
\lim _{\lambda \rightarrow e_{i}^{-}} g^{-}(x)=-\infty, \lim _{\lambda \rightarrow e_{i}^{+}} g^{-}(x)=\infty, \quad i=1,2, \ldots, N-1 .
$$

We next show that $g^{+}(\lambda)$ and $g^{-}(\lambda)$ do not intersect with each other. To this end, we set

$$
e(s):=\frac{s^{2 N+1}-s^{2 N-1}}{s^{4 N}-1} .
$$

If $s^{2 N+1}-s^{2 N-1}=0$, then $s^{2}=1$. Now,

$$
\lim _{s \rightarrow 1} e(s)=\lim _{s \rightarrow 1} \frac{(2 N+1) s^{2 N}-(2 N-1) s^{2 N}}{4 N s^{4 N-1}}=\frac{1}{2 N} .
$$

Similarly,

$$
\lim _{s \rightarrow 1^{-}} e(s)=-\frac{1}{2 N}
$$




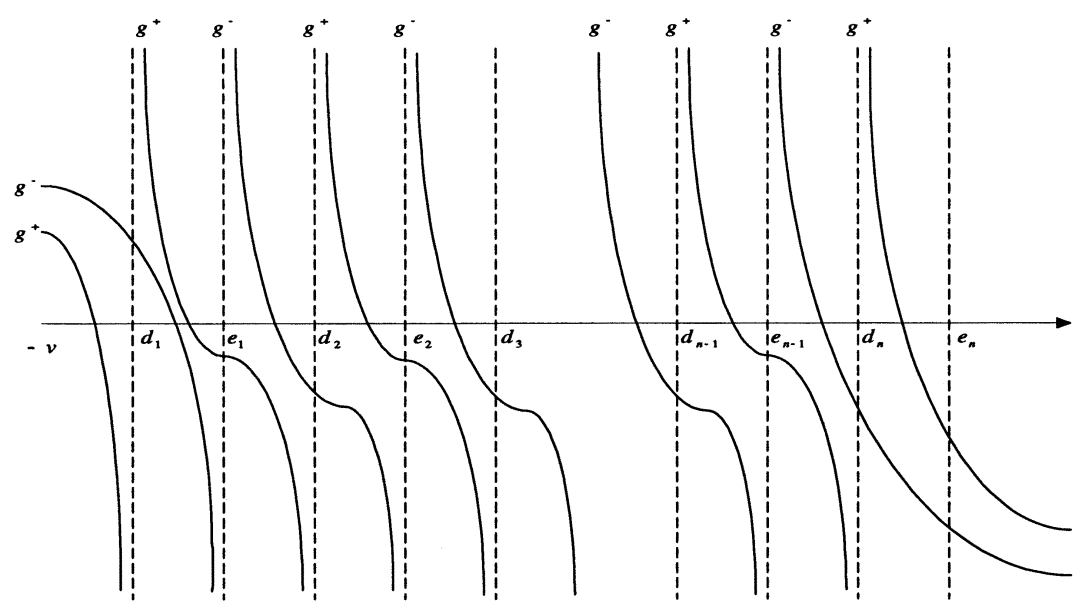

FIG. 1.

Thus, $e(s)$ is nonzero for all $\lambda$. It then follows from Proposition 2.2, equation (2.8), and the fact that $g^{+}(\lambda)$ does not intersect with $g^{-}(\lambda)$ for all $\lambda$ that the assertions of the proposition hold true.

Using the assertions of Proposition 2.8, we give a rough drawing of $g^{+}(\lambda)$ and $g^{-}(\lambda)$. Figure 1 reflects only the information of $g^{ \pm}(\lambda)$ obtained in Proposition 2.8. It is by no means an accurate drawing of the graphs of $g^{ \pm}(\lambda)$.

We are now ready to state our main results.

Notation 2.2. Set $S=\{-v<\lambda<0: \quad \lambda$ is the symmetric eigenvalue of system (1.2a)-(1.2h) $\}$, and $S_{a}=\{-v<\lambda<0: \quad \lambda$ is the antisymmetric eigenvalue of system (1.2a)-(1.2h) $\}$. The cardinalities of $S$ and $S_{a}$ are denoted by $\#_{s}$ and \# $\#_{s a}$, respectively. The sum of $\#_{s}$ and $\#_{s a}$ is denoted by \#.

Theorem 2.1. Let $2>\alpha>0$. Suppose $(\gamma, \beta) \in R_{1} \bigcup R_{2}$ and $v>\frac{2}{m^{\prime}}$. Then $\#_{s}=N+1$ and $\#_{a s}=N$.

Proof. Using Proposition 2.6, we see that $f^{ \pm}(-v)<g^{ \pm}(-v)$. Thus, all eigenvalues of the system are greater than $-v$. It also follows from Proposition 2.6 that $f^{ \pm}(0) \geq$ $\frac{\alpha^{\prime}}{\alpha+1}>\frac{\alpha^{\prime}-2}{\alpha}=g^{ \pm}(0)$. Hence, we conclude, via Figure 1 , that $\# \geq 2 N+1$. However, the other eigenvalues of the system comes from the intersection of $f^{ \pm}(\lambda)$ and $g^{ \pm}(\lambda)$ on $\left(0, \frac{2}{m}\right)$. Upon using the fact that $f^{ \pm}(0) \geq g^{ \pm}(0)$, we conclude that $\# \leq 2 N+1$. Hence $\#=2 N+1$. It is clear from Figure 1 that the assertions of the theorem hold.

Remark 2.1 .

1. Theorem 2.1 implies that if the depth of the well is "sufficiently large," then all the energy levels fall in the well.

2. Using Propositions 2.6 and 2.7, one also sees that for Neumann boundary conditions $(\gamma=1, \beta=0)$, at least one energy level falls in the well regardless of the size of the well.

TheOrEm 2.2. Let $(\gamma, \beta) \in R_{1}, \beta \neq 0$, and $v \leq \frac{2}{m^{\prime}}$. If $e_{k}<0<d_{k+1}$ for some $1 \leq k \leq N$, the following table holds true:

\begin{tabular}{c|c|c|c}
\hline & $f^{+}(0)>g^{-}(0)$ & $f^{+}(0)>g^{+}(0)$ & \\
$f^{-}(0)<g^{-}(0)$ & $g^{+}(0)>f^{-}(0)$ \\
\hline$\#_{s}$ & $k+1$ & $k+1$ & $k$ \\
\hline$\#_{a s}$ & $k+1$ & $k$ & $k$ \\
\hline
\end{tabular}


If $d_{k}<0<e_{k}$ for some $1 \leq k \leq N$, the following table holds true:

\begin{tabular}{c|c|c|c}
\hline & $f^{+}(0)>g^{+}(0)$ & $f^{-}(0)>g^{-}(0)$ & \\
$g^{+}(0)>f^{+}(0)$ & $f^{-}(0)<g^{-}(0)$ \\
\hline$\#_{s}$ & $k+1$ & $k$ & $k$ \\
\hline$\#_{a s}$ & $k$ & $k$ & $k-1$ \\
\hline
\end{tabular}

If $e_{k}<0=d_{k+1}$ for some $1 \leq k \leq N$, the following table holds true:

\begin{tabular}{c|c|c}
\hline & $f^{-}(0)>g^{-}(0)$ & $f^{-}(0)<g^{-}(0)$ \\
\hline$\#_{s}$ & $k+1$ & $k+1$ \\
\hline$\#_{a s}$ & $k+1$ & $k$ \\
\hline
\end{tabular}

If $d_{k}<0=e_{k}$ for some $1 \leq k \leq N$, then the following table holds true:

\begin{tabular}{c|c|c}
\hline & $f^{+}(0)>g^{+}(0)$ & $f^{+}(0)<g^{+}(0)$ \\
\hline$\#_{s}$ & $k+1$ & $k$ \\
\hline$\#_{a s}$ & $k$ & $k$ \\
\hline
\end{tabular}

Proof. We first note that if $(\gamma, \beta) \in R_{1}$ and $\beta \neq 0$, then $f^{+}(\lambda)>f^{-}(\lambda)$ on $(-\infty, 0]$. The assertions of the theorem now follow from Propositions 2.2, 2.4, 2.8 and Figure 1.

For $(\gamma, \beta) \in R_{2}$ or $\beta=0$, similar tables as above can be obtained. In each of the tables above, the largest numbers of $\#_{s}$ and $\#_{a s}$ occur when $f^{ \pm}(0)>g^{ \pm}(0)$. We next show that this is the case when the length $v$ of the well is sufficiently close to $\frac{2}{m^{\prime}}$.

TheOrem 2.3. Let $m \approx m^{\prime}$ and $(\gamma, \beta) \in R_{1}$. Suppose $v \leq \frac{2}{m^{\prime}}$ and $v$ is sufficiently close to $\frac{2}{m^{\prime}}$. Then $f^{ \pm}(0)>g^{ \pm}(0)$. Consequently, only the second column of each table in Theorem 2.2 holds.

Proof. Direct calculation would yield that

$$
g^{+}\left(e_{k}\right)=\frac{\alpha^{\prime}-1}{\alpha}+\frac{1}{\alpha} \cos \frac{k \pi}{N}
$$

and

$$
g^{-}\left(d_{k}\right)=\frac{\alpha^{\prime}-1}{\alpha}+\frac{1}{\alpha} \sin \frac{(2 k-1) \pi}{2 N} .
$$

Suppose $v\left(\leq \frac{2}{m^{\prime}}\right)$ is sufficiently close to $\frac{2}{m^{\prime}}$. Then $e_{k}, d_{k} \geq 0$ only if $k$ is sufficiently large. If $k$ is large, then $g^{+}\left(e_{k-1}\right)<0$ and $g^{-}\left(d_{k-1}\right) \approx 0$, where we may assume that $d_{k-1}, e_{k-1}<0$. Since $g^{ \pm}$are decreasing, $g^{+}(0)<g^{+}\left(e_{k-1}\right)<0$ and $g^{-}(0)<$ $g^{-}\left(d_{k-1}\right) \approx 0$. However, we see, via Proposition 2.6(i), that $f^{ \pm}(0)=\frac{\alpha^{\prime}}{\alpha+1}>0$. We thus complete the proof of the theorem.

We conclude the paper by mentioning some possible future related work. First, the study of discrete higher-dimensional Schrödinger problems is of considerable interest. Second, it would also be of interest to study a continuous version of the problem described in this paper. Finally, though the calculations would get more complicated, our approach here can be used to treat nonsymmetric boundary conditions.

\section{REFERENCES}

[1] M. S. Ashbaugh And R. D. Benguria, Optimal bounds for ratios of eigenvalues of onedimensional Schrödinger operators with Dirichlet boundary conditions and positive potentials, Comm. Math. Phys., 124 (1989), pp. 403-415. 
[2] M. S. Ashbaugh and R. D. Benguria, Eigenvalue ratios for Sturm-Liouville operators, J. Differential Equations, 103 (1993), pp. 205-219.

[3] R. Courant and D. Hilbert, Methods of Mathematical Physics, Vol. 1, Wiley, New York, 1990.

[4] G. H. Golub, Some modified matrix eigenvalue problems, SIAM Rev., 15 (1973), pp. 318-334.

[5] Y. L. HuAng AND C. K. LAW, Eigenvalue ratios for the regular Sturm-Liouville system, Proc. Amer. Math. Soc., 124 (1996), pp. 1427-1436.

[6] C. Juang, K. J. Kuhn, and R. B. Darling, Stark shift and field-induced tunneling in $A l_{\mathrm{x}} G a_{1-x} A s / G a A s$ quantum-well structures, Phys. Rev. B, 41 (1990), pp. 12047-12053.

[7] C. JuAng, J. Y. Wang, AND J. JuAng, Controlling chaotic behavior of heavy to light hole mixing tunneling by external electric fields, IEEE J. Quantum Electron., 33 (1997), pp. 13451349.

[8] S. LURYI, Polarization oscillations in coupled quantum wells-scheme for generation of submillimeter electromagnetic waves, IEEE J. Quantum Electron., 27 (1991), pp. 54-60.

[9] B. N. Parlett And T. T. Lu, Minimum eigenvalue separation, Linear Algebra Appl., to appear.

[10] I. M. Singer, B. Wong, S.-T. Yau, And S. S.-T. Yau, An estimate of the gap of the first two eigenvalues in the Schrödinger operator, Ann. Scuola. Norm. Sup. Pisa Cl. Sci. (4), 12 (1985), pp. 319-333. 